\title{
AN ANATOMIC RECONSTRUCTION OF THE DISTAL RADIOULNAR JOINT FOR POSTTRAUMATIC INSTABILITY BY ADAMS-BERGER PROCEDURE
}

\author{
Georgi Ganchev \\ Department of Orthopedics and Traumatology, Faculty of Medicine, \\ Medical University of Varna
}

\begin{abstract}
INTRODUCTION: The distal radio ulnar joint has an important and complex role in the motion of the wrist joint and the entire upper limb. The anatomical relations between the distal radius, ulna and the ulnar carpus are very fine and precise and even minor disturbances in their congruence may lead to significant changes in motions and pronounced pain syndrome. The post-traumatic instability of the distal radio ulnar joint (DRUJ) affects all age groups. However, the contingent of professional athletes may be determined as the risk one. DRUJ instability resulting from a trauma can lead to significant discomfort expressed in ulnar pain syndrome involving the ulnocarpal joint, weakness and limitation of the motion of the radiocarpal joint.
\end{abstract}

AIM: The aim of this paper is to present the Adams-Berger surgical procedure and the results after the anatomical reconstruction in cases of DRUJ instability.

MATERIALS AND METHODS: During the period 2009 - 2015, at the Clinic of Orthopedics and Traumatology at St. Anna University Hospital for Active Treatment, Varna, 17 patients with DRUJ instability were operated on by the Adams procedure.

RESULTS: DRUJ stability was observed three years after the surgery on average and 1st degree instability was seen in one of the patients. The average Modified Mayo Wrist Score was 70.01 points prior to the surgery and 93.6 points at the $36^{\text {th }}$ month after the surgery. The subjective opinion of 14 of the patients was that the result was excellent, two of them determined it as very good and one of them - as good. In all of the patients an improvement in a number of parameters was observed, such as flexion, extension, radial and ulnar deviation, pronosupination and muscle strength reaching values comparable to those in the health hand.

DISCUSSION: There are many surgical techniques aimed at restoring DRUJ stability. An alternative option is ligamentoplasty with half of the tendon of flexor capri ulnaris, which, however, does not give absolute stability and has limited indications. In the presence of malposition after distal radius fracture, the stability can be improved after corrective osteotomy.

Address for correspondence:

Georgi Ganchev

Faculty of Medicine

Medical University of Varna

55 Marin Drinov St

9002 Varna

e-mail:dr.ganchev@hotmail.com

Received: April 24, 2017

Accepted: June 23, 2017
CONCLUSION: Ligament reconstruction is an effective method for treating post-traumatic DRUJ instability. In the case of non-deformity of the radius and arthritis of the DRUJ, Adams technique is a method of choice for restoring radial distal stability.

Keywords: distal radio ulnar joint (DRUJ), triangular fibrocartilage complex (TFCC), instability, Adams - Berger procedure 


\section{INTRODUCTION}

The distal radio ulnar joint has an important and complex role in the motion of the wrist joint and the entire upper limb. The anatomical relations between the distal radius, ulna and the ulnar carpus are very fine and precise and even minor disturbances in their congruence may lead to significant changes in motion and pronounced pain syndrome.

The post-traumatic instability of the distal radio ulnar joint (DRUJ) affects all age groups and the contingent of professional athletes may be determined as the risk one.

DRUJ instability resulting from a trauma can lead to a significant discomfort expressed in ulnar pain syndrome involving the ulnocarpal joint, weakness and limitation of the motion of the radiocarpal joint.

The specific biomechanics and kinematics of DRUJ determine it as unstable by nature. The posttraumatic, acute and chronic instability requires a precise diagnosis predetermining the algorithm of treatment.

The most common cause for DRUJ instability usually is a result of trauma. The most common traumas leading to instability are distal fractures of the radius and the forearm, isolated fractures of the styloid process of the ulna, Galeazzi fracture-dislocation and injuries occurring in hyperpronation and extension of the wrist joint causing damage to the triangular fibrocartilage complex (TFCC). DRUJ instability is characterized by volar and dorsal subluxation of the ulnar head, loss of rotation, and characteristic ulnocarpal pain. DRUJ instability treatment is conducted exclusively by surgical management. There are various types of surgical options consistent with the degree of TFCC injury, the difference in the length of the ulna and radius, and the presence or absence of DRUJ arthrotic changes. Surgical techniques vary from partial resection of the ulnar head, distal radioulnar arthrodesis, and TFCC suture to anatomical reconstruction and stabilization of the joint. Soft-tissue reconstructive procedures for DRUJ stabilization are indicated in the cases when TFCC cannot be restored. There are techniques for indirect repair of the stability, such as tenodesis using the flexor carpi ulnaris or extensor carpi ulnaris tendons (1). These non-anatomical methods cannot al- ways fully restore the mobility of DRUJ, so anatomical techniques are preferred. In 2002, Adams и Berger (2) reported the clinical results from a DRUJ recovery technique by using a free tendon graft in cases where TFCC cannot be recovered.

\section{AIM}

The aim of this paper is to present the Adams-Berger surgical procedure and the results after the anatomical reconstruction in cases of DRUJ instability.

\section{MATERIALS AND METHODS}

During the period 2009 - 2015, at the Clinic of Orthopedics and Traumatology at St. Anna University Hospital for Active Treatment, Varna, 17 patients with DRUJ instability were operated on by the Adams procedure. All patients were classified as 4 th degree by the Classification of Mathoulin, where a tear of the proximal and distal components of TFCC was observed, with no recovery potential (Table 1).

Indications for Adams-Berger DRUJ stabilization:

$\diamond 4^{\text {th }}$ degree DRUJ instability according to $\mathrm{Ma}-$ thoulin (Fig.1);

$\diamond$ no possibility to perform arthroscopic or open reconstruction of the TFCC, due to poor healing potential;

$\diamond$ persisting DRUJ instability after ulnar shortening osteotomy.

To establish an accurate diagnosis and conduct the appropriate treatment, radiography was performed in all patients. It showed dorsal subluxation of the ulnar head. Four patients underwent arthroscopy and in 7, MRI was conducted (Fig. 1).

All patients were operated on using the procedure described by Adams-Berger (2) - anatomical reconstruction of DRUJ with a free tendon graft of palmaris longus or an artificial transplant (Fig. 2).

Surgical technique:

$\diamond$ In all patients an arthroscopy of the wrist joint and the DRUJ were performed to confirm the lack of possibility to perform arthroscopic or open repair of TFCC.

$\diamond$ Dorsal incision of 5-8 $\mathrm{cm}$ between the $5^{\text {th }}$ and the 6th osteofibrous channels (compartments) was made. 
Table 1. Classification of Mathoulin

\begin{tabular}{|c|c|c|c|c|c|c|}
\hline \multirow{2}{*}{ 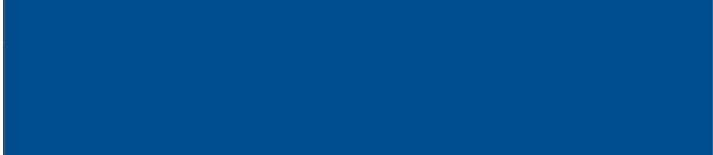 } & \multirow{2}{*}{$\begin{array}{l}\text { Clinically } \\
\text { manifested } \\
\text { DRUJ } \\
\text { instability }\end{array}$} & \multicolumn{2}{|c|}{ TFCC Lesion } & \multirow[b]{2}{*}{$\begin{array}{l}\text { Trampo- } \\
1 \text { line effect }\end{array}$} & \multirow[b]{2}{*}{$\begin{array}{c}\text { Hook } \\
\text { Test }\end{array}$} & \multirow[b]{2}{*}{$\begin{array}{c}\text { Ghost } \\
\text { Test }\end{array}$} \\
\hline & & Distal & Proximal & & & \\
\hline
\end{tabular}

Degree 1 Repairable distal zone of TFCC

Repairable

Degree 2 complete lesion of TFCC

Repairable

Degree 3 proximal zone of TFCC

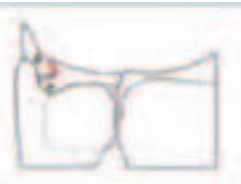

Weak or absent Lesion Intact

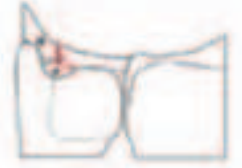

Medium or severe

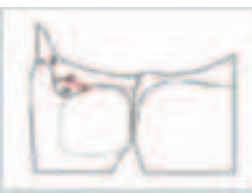
Medium or
severe

Intact Lesion

Severe

Lesion

Lesion

Medium or severe

Degree 5 DRUJ arthrosis

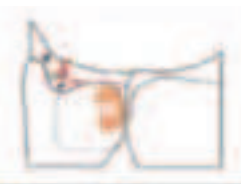

$\diamond$ The fifth osteofibrous channel was opened and the extensor tendon of the fifth finger was retracted radially thus visualizing the articular capsule of DRUJ.
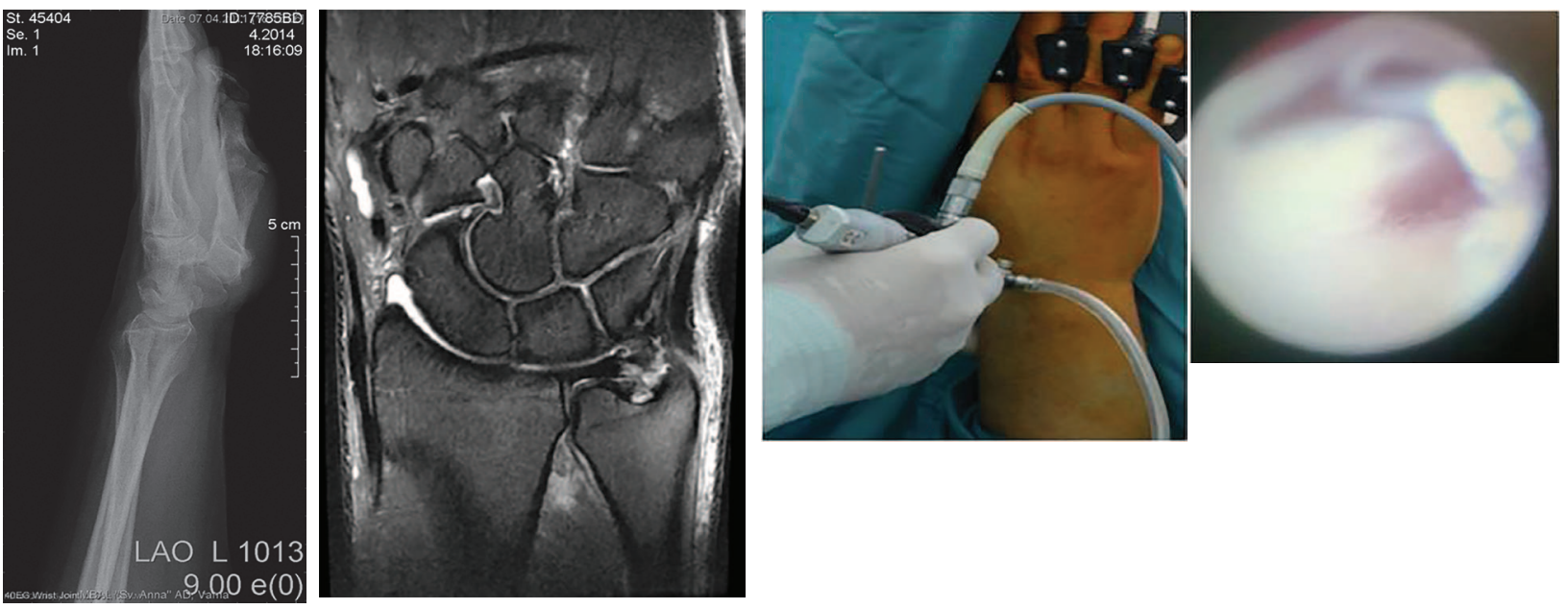

Fig. 1. A - X-ray image; $B-M R I$; $C$-Arthroscopy. 

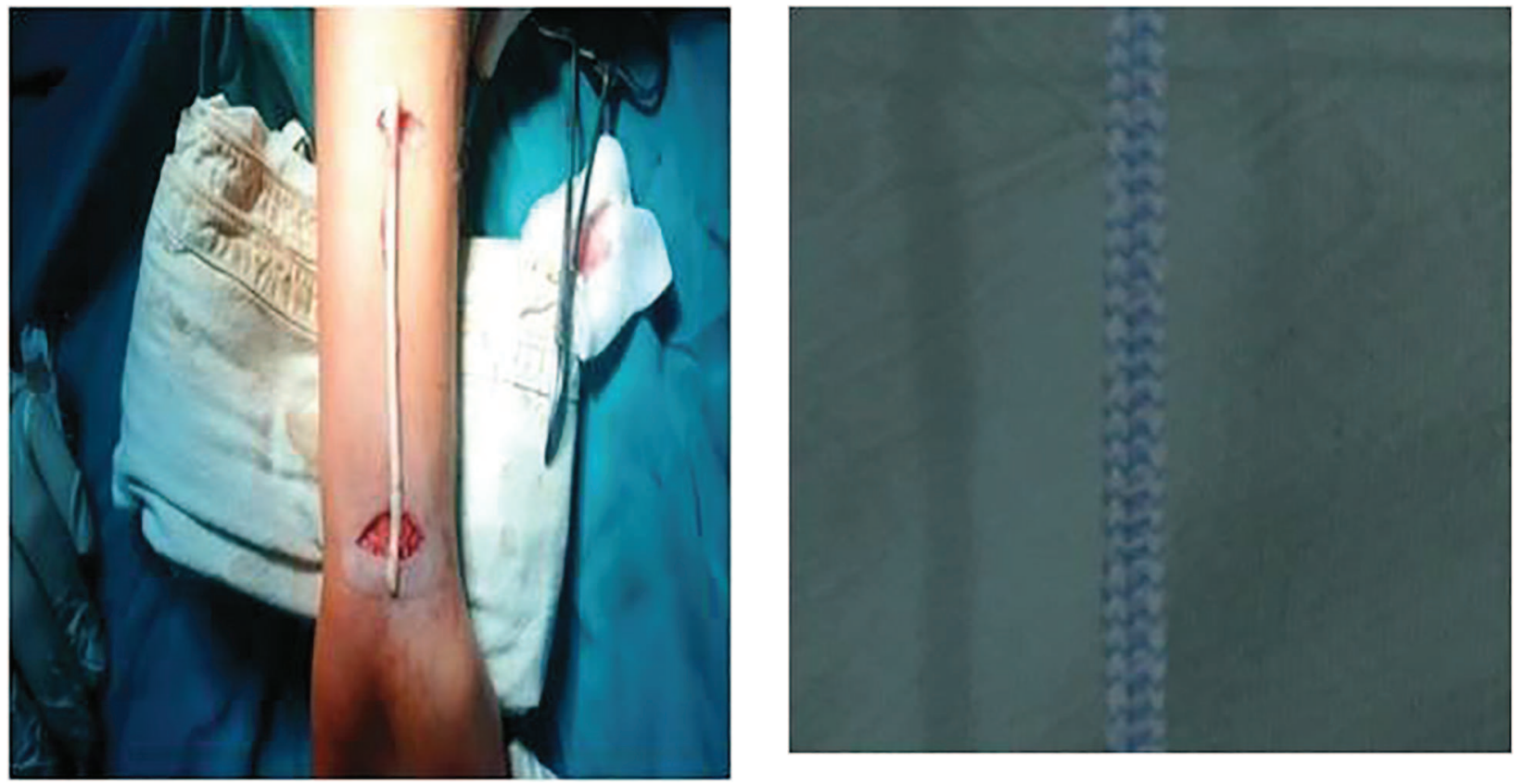

Fig. 2. Tendon graft (A-palmaris longus, B-artificial transplant)

the tunnel to accommodate the graft $-5 \mathrm{~mm}$ proximal to the lunate fossa and $5 \mathrm{~mm}$ radial from the ulnar rim of the radius (Fig. 3).

$\diamond$ A guide wire for a $5 \mathrm{~mm}$ cannulated drill bit is driven into the base of the styloid process of the ulna, between the fovea and the neck of the

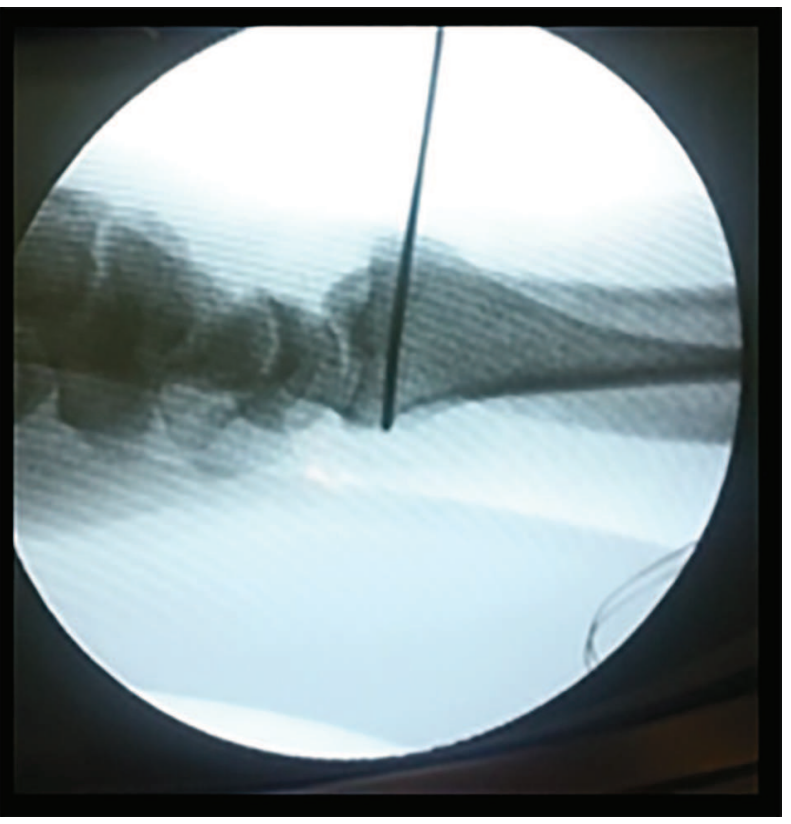

bone, to prepare a tunnel. If necessary, the tunnel may be enlarged (Fig. 4).

$\diamond$ The graft was placed through the radial tunnel (Fig. 5), (Fig. 7).

The graft was placed through the ulnar tunnel and tightened until DRUJ was stabilized (Fig. 6), (Fig. 7).

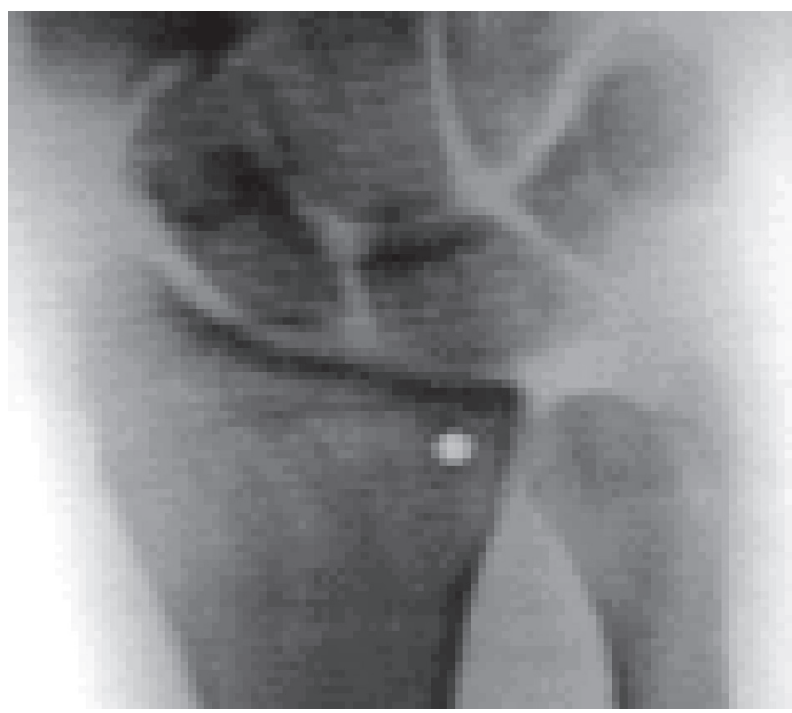

Fig. 3. Tunnel in the distal radius for the tendon graft 


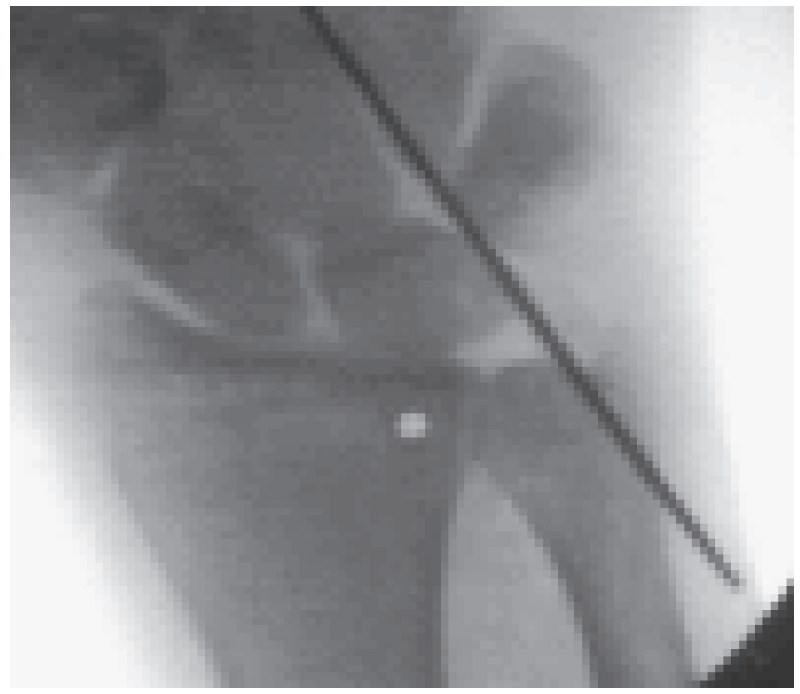

Fig. 4. Insertion of the guide wire into the drilled tunnel for the tendon graft

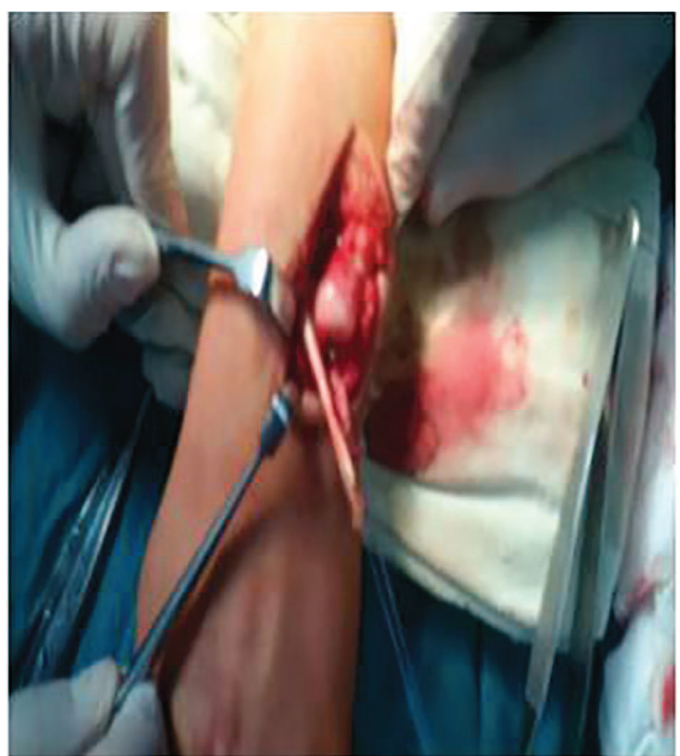

Fig. 5 Positioning of the tendon of the palmaris longus through the tunnel of the distal radiust

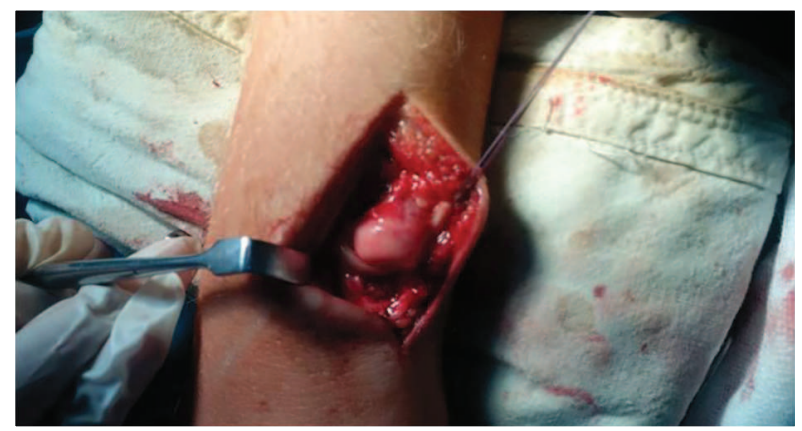

Fig. 6. DRUJ stabilization after graft tightening

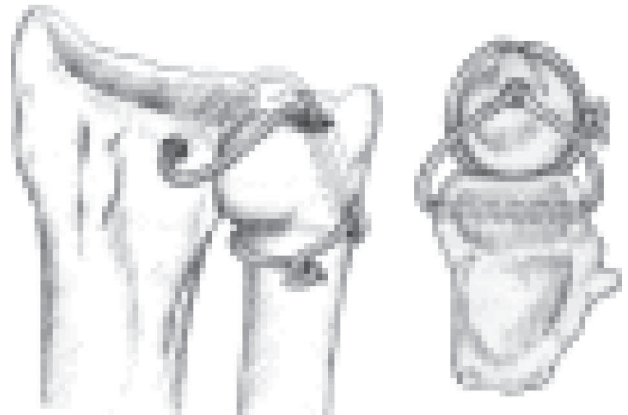

Fig. 7. Passing of the graft through the bone tunnels of the radius and the ulna - illustration (Source: Green's Operative Hand Surgery, $5^{\text {th }}$ edition. Philadelphia: Churchill Livingstone; 2005)

\section{RESULTS}

DRUJ stability was observed three years after the surgery on average and $1^{\text {st }}$ degree instability was seen in one of the patients. The average Modified Mayo Wrist Score was 70.01 points prior to the surgery and 93.6 points at the $36^{\text {th }}$ month after the surgery. The subjective opinion of 14 of the patients was that the result was excellent, two of them determined it as very good and one of them - as good. In all of the patients, an improvement in a number of parameters was observed, such as flexion, extension, radial and ulnar deviation, pronosupination and muscle strength reaching values comparable to those in the healthy hand (Table 2).

\section{DISCUSSION}

There are many surgical techniques aimed at restoring DRUJ stability. An alternative option is ligamentoplasty with half of the tendon of flexor capri ulnaris, which, however, does not give absolute stability and has limited indications. In the presence of malposition after distal radius fracture, the stability can be improved after corrective osteotomy.

Adams procedure allows the anatomical reconstruction of TFCC and restoration of the stability, but the method is indicated for patients with $4^{\text {th }}$ degree injury by Mathoulin, without presence of arthrotic changes and ulnoradial mismatch, which makes the diagnostics a primary success factor. 
An Anatomic Reconstruction of the Distal Radioulnar Joint for Posttraumatic Instability by Adams-Berger Procedure

Table 2. Dynamics of various functional parameters prior to and after the surgical management and the values of the contralateral healthy hand

\section{Preoperative value}

Postoperative value

Healthy hand

Flexion

$52^{0}$

Extension

$64^{0}$

$64^{0}$

$67^{0}$

Radial deviation

$20^{\circ}$

$71^{0}$

$73^{0}$

Ulnar deviation

$30^{\circ}$

$27^{0}$

$28^{0}$

Pronosupination

$0-172^{0}$

$37^{0}$

$38^{0}$

Muscle strength

$22^{0}$

$0-178^{0}$

$0-179^{0}$

$35^{0}$

$38^{0}$

\section{CONCLUSION}

Ligament reconstruction is an effective method for treating post-traumatic DRUJ instability. In the case of non-deformity of the radius and arthritis of DRUJ, the Adams technique is a method of choice for restoring radial distal stability. The exact assessment of the indications consistent with the choice of an adequate surgical technique is crucial to achieve a good functional result. The Adams-Berger procedure for anatomical DRUJ reconstruction is an effective procedure for chronic post-traumatic instability.

\section{REFERENCES}

1. Breen TF, Jupiter JB. Extensor carpi ulnaris and flexor carpi ulnaris tenodesis of the unstable distal ulna. J Hand Surg Am. 1989;14(4):612-7.

2. Adams BD, Berger RA. An anatomic reconstruction of the distal radioulnar ligaments for posttraumatic distal radioulnar joint instability. J Hand Surg Am. 2002;27(2):243-51.

3. Mathoulin $\mathrm{Ch}$, Atzei A. Réparation du ligament triangulaire (TFCC) : rapport d'une série de 72 cas suivant une nouvelle classification; e-mémoires de l'Académie Nationale de Chirurgie, 2010, 9 (1): 21-7.

4. Kihara H, Short WH, Werner FW, Fortino MD, Palmer AK. The stabilizing mechanism of the distal radioulnar joint during pronation and supination. J Hand Surg Am. 1995;20(6):930-6. doi: 10.1016/S0363-5023(05)80139-X

5. Oda $\mathrm{T}$, Wada $\mathrm{T}$, Isogai $\mathrm{S}$, Iba $\mathrm{K}$, Aoki M, Yamashita

T. Corrective osteotomy for volar instability of the distal radioulnar Joint associated with radial shaft malunion. J Hand Surg Eur Vol. 2007;32(5):573-7.

dio: 10.1016/J.JHSE.2007.04.018 